\title{
Relationship between the Magnetic Flux of Solar Eruptions and the Ap Index of Geomagnetic Storms
}

\author{
I.M. Chertok ${ }^{1}$ - M.A. Abunina ${ }^{1}$. \\ A.A. Abunin ${ }^{1}$ - A.V. Belov ${ }^{1}$. \\ V.V. Grechnev ${ }^{2}$
}

(C) Springer $\bullet \bullet \bullet \bullet$

\begin{abstract}
Solar coronal mass ejections (CMEs) are main drivers of the most powerful non-recurrent geomagnetic storms. In the extreme-ultraviolet range, CMEs are accompanied by bright post-eruption arcades and dark dimmings. The analysis of events of the Solar Cycle 23 (Chertok et al., 2013, Solar Phys. $\mathbf{2 8 2}, 175)$ revealed that the summarized unsigned magnetic flux in the arcades and dimming regions at the photospheric level, $\Phi$, is significantly related to the intensity (Dst index) of geomagnetic storms. This provides the basis for the earliest diagnosis of geoefficiency of solar eruptions. In the present article, using the same data set, we find that a noticeable correlation exists also between the eruptive magnetic flux, $\Phi$, and another geomagnetic index, Ap. As the magnetic flux increases from tens to $\approx 500$ (in units of $10^{20} \mathrm{Mx}$ ), the geomagnetic storm intensity measured by the 3 -hour Ap index, enhances in average from Ap $\approx 50$ to a formally maximum value of 400 (in units of $2 \mathrm{nT}$ ). The established relationship shows that in fact the real value of the Ap index is not limited and during the most severe magnetic storms may significantly exceed 400.
\end{abstract}

Keywords: Solar eruptions; Arcades; Coronal dimming; Coronal mass ejections; Magnetic fields; Geomagnetic storms

\section{Introduction}

Intense non-recurrent geomagnetic storms (GMSs) are the most significant space weather disturbances. In contrast to relatively weak recurrent storms associated with high-speed solar wind streams from coronal holes, they are initiated by coronal mass ejections (CMEs) and their interplanetary extensions (ICMEs) (e.g., Bothmer and Zhukov, 2007, Gopalswamy, 2009). A GMS occurs when a large and fast CME/ICME brings to the Earth the magnetic field with a

\footnotetext{
${ }^{1}$ Pushkov Institute of Terrestrial Magnetism, Ionosphere and Radio Wave Propagation (IZMIRAN), Troitsk, Moscow

Region, 142190 Russia email: ichertok@izmiran.ru

${ }^{2}$ Institute of Solar-Terrestrial Physics SB RAS, Lermontov

St. 126A, Irkutsk 664033, Russia email: grechnev@iszf.irk.ru
} 
sufficiently strong and prolonged southward (negative) $B_{z}$ component. One of the most important tasks of the solar-terrestrial physics and space weather prediction is diagnostics of geoeffectiveness of CMEs, i.e., quantitative forecast of a possible non-recurrent GMS from observed characteristics of the eruption that just occurred. Existing algorithms of such diagnostics are based in one way or another on the measurements of the CME speed and shape in the plane of the sky near the Sun (Gopalswamy, 2009, Kim et al., 2010).

We develop another approach to the early diagnostics of solar eruptions, in which quantitative characteristics of such large-scale CME manifestations as post-eruption (PE) arcades and dimmings observed in the extreme ultraviolet (EUV) range are used as key parameters instead of the projected CME speed and shape (Chertok and Grechnev, 2006 Chertok et al., 2013). Dimmings are CMEassociated regions in which the EUV (and soft X-ray) brightness of coronal structures is temporarily reduced during an ejection and persists over many hours (Thompson et al., 1998: Webb et al., 2000: Hudson and Cliver, 2001). The deepest stationary long-lived dimmings adjacent to the eruption center are interpreted mainly as a result of plasma outflow and density decrease in footpoints of erupting and expanding CME flux ropes. Large-scale arcades of bright loops enlarging in size over time arise at the place of the main body of pre-eruption magnetic flux ropes ejected as CMEs (Kahler, 1977: Sterling et al., 2000: Tripathi, Bothmer, and Cremades, 2004 As a whole, $\mathrm{PE}$ arcades and dimmings visualize the structures and areas involved in the processes of CME eruptions.

A number of studies indicate that parameters of CMEs and near-Earth magnetic clouds (MCs) are governed by total magnetic fluxes in their solar source regions. Qiu and Yurchyshyn (2005) found a correlation between the CME speed and the total reconnection flux in associated flares. Leamon et al. (2004) and Mandrini et al. (2005) demonstrated that the total magnetic fluxes in MCs estimated from in situ measurements near the Earth and their solar sources were close to each other. Webb et al. (2000) and Attrill et al. (2006) found a similarity between the magnetic fluxes in MCs and dimming regions. Qiu et al. (2007) and $\mathrm{Hu}$ et al. (2014) found this similarity for the magnetic reconnection flux in several events. Therefore, a correspondence is expected between the magnetic flux involved in an eruption, on the one hand, and parameters of the associated geospace disturbances, on the other hand. If a magnetic cloud carries a negative $B_{z}$ component, then a correlation might be expected between the magnetic fluxes in eruption regions and the intensities of the GMSs that they produce.

Chertok et al. (2013) established that a statistical dependence of the GMS intensity measured by the Dst index (as well as of the onset and peak GMS transit times and Forbush decrease magnitudes) on the eruptive magnetic flux in the arcades and dimming regions, $\Phi$, exists indeed. The aim of the present work is to analyze the relationship between this eruptive flux $\Phi$ and another geomagnetic index Ap that also characterizes the GMS intensity and is often used in the solar-terrestrial forecasting. 


\section{Analyzed Parameters and Data}

Recall that the hourly storm-time disturbance index Dst (see http://wdc.kugi.kyoto-u.ac.jp/dstdir/index.html is calculated from data of four low-latitude geomagnetic observatories and characterizes the effect of the global equatorial ring current. The latter is manifested in the inner magnetosphere and causes a decrease of the horizontal component of the terrestrial magnetic field during the main phase of GMSs. The linear planetary 3-hour Ap index, considered below, characterizes the strength of auroral currents. The Ap index is defined as the mean value of the variations of the terrestrial magnetic field, which corresponds to a logarithmic Kp index, measured by data of 13 geomagnetic stations located at moderately high geomagnetic latitudes mainly in the northern hemisphere (Siebert and Meyer, 1996. Zabolotnaya, 2007). The Ap index varies from 0 to a formally maximum value of 400 corresponding to $\mathrm{Kp}=9$, and is measured in units of $2 \mathrm{nT}$ (hereinafter, as is often done, we will omit the units). We emphasize that the formal upper limit of $\mathrm{Ap}=400$ is is a condition that appears as a result of the conversion of Ap from Kp indices. In a physical sense the Ap index is not limited. It is determined by real variations of the terrestrial magnetic field components and during the strongest magnetic storms can considerably exceed 400. Features of the Dst and Ap indices are such that relationships between them in concrete events may be different, depending on the impact of an ICME on the equatorial and auroral current systems. In addition, the peak time of the Dst and Ap indices are not always identical and may differ by a few hours.

We consider the same ensemble of the events that has been analyzed by Chertok et al. (2013): strong non-recurrent GMSs during Solar Cycle 23 (19962008) with Dst $\leq-100 \mathrm{nT}$, which reliably or with a high probability are identified with a concrete eruption from the central zone of the visible solar hemisphere within $\pm 45^{\circ}$ from the central meridian. We will distinguish events caused by eruptions that occurred in active regions (ARs), and events associated with filament eruptions outside ARs referring to them as "AR events" and "non-AR events", respectively. These two categories of events differ significantly in the characteristics of accompanying arcades and dimmings, properties of CMEs/ICMEs, and intensity of GMSs that they cause (e.g., Švestka, 2001).

Our source data are the EUV solar images obtained with the Extreme ultraviolet Imaging Telescope (EIT: Delaboudinière et al., 1995) in the $195 \AA$ channel and the magnetograms obtained with the Michelson Doppler Imager (MDI: Scherrer et al., 1995), both on the Solar and Heliospheric Observatory (SOHO: Domingo, Fleck, and Poland, 1995). For each of the analyzed events, after a routine processing of corresponding FITS files, the solar rotation in the analyzed images was compensated, and then the same fixed image before an eruption was subtracted from all subsequent ones to obtain fixed-base difference images (Chertok and Grechnev, 2005). Significant regions of the arcades and dimmings are extracted following selected relative (not absolute) criteria of brightness variations. The analysis showed that a brightness depression of more than $40 \%$ from the pre-eruption level was an optimal criterion for extraction of relevant significant dimmings. For PE arcades, an appropriate criterion was that which extracted the area around the eruption center where the brightness in the 
$195 \AA$ channel exceeded $5 \%$ of the maximum one. A total (unsigned) eruptive magnetic flux within the extracted arcade and dimming areas was evaluated at the photospheric level by co-aligning the resulting EIT difference images with an MDI line-of-sight magnetogram recorded just before an eruption. The detailed description of all required procedures is given in Chertok et al. (2013).

In this case we will characterize the maximum intensity of GMSs by a peak in the 3-hour Ap index, the values of which are determined in the GeoForschungsZentrum (GFZ), Potsdam, and presented at ftp://ftp.gfz-potsdam.de/pub/home/obs/kp-ap/wdc/ The comprehensive list of the analyzed events and their parameters are shown in a table displayed at the site http://www.izmiran.ru/ ichertok/Ap/. The table includes 90 events and contains in particular the following data: the numbers of the GMS events according to the Coordinated Data Analysis Workshop (CDAW) catalog (Zhang et al., 2007); information on date, time, and intensity of a GMS, including Ap index; data about the eruption, which reliably or with a high probability was a source of the given GMS; the total magnetic fluxes in the arcades and dimming regions; and some others.

\section{Results}

First, we consider the events, in which a GMS is reliably identified with a particular solar eruption. Figure $1 \mathrm{H}$ shows the location of such events on the plot of the geomagnetic Ap index vs. the eruptive magnetic flux in the arcades and dimming regions, $\Phi$ ". In this figure and the following ones, the diamonds denote the AR eruptions, and the triangles correspond to the non-AR eruptions.

As noted by Chertok et al. (2013), few events associated with filament eruptions outside ARs (non-AR events) form a separate group, which is markedly different from the events produced by the eruptions in ARs (AR events). The non-AR events are characterized by relatively low magnetic fluxes, $\Phi<80 \times$ $10^{20} \mathrm{Mx}$, but they can cause GMSs with Ap $>200$. Figure 1 does not show any pronounced dependence of Ap on $\Phi$ for such events. A possible reason for these features of the non-AR events is that the criteria for the extraction of significant arcades and dimmings accepted for the AR events are not entirely suitable for the non-AR events. It is known (Švestka, 2001) that filament eruptions outside ARs are accompanied by relatively weak, but more extensive PE arcades, as well as by shallower dimmings in comparison with eruptions in ARs. This suggests that the relative thresholds of the brightness variations for the extraction of the arcades and dimmings indicated above should be optimized at lower levels for the non-AR events. In this case, the values of the eruptive magnetic flux would noticeably increase, but in different ways for different events. Some other factors may also affect the characteristics of GMSs caused by the non-AR eruptions. Therefore, the non-AR events require a special additional analysis and will not be counted below in this paper.

Among the AR events (diamonds), attention should be paid to an event that strongly deviates from other GMSs. This is a famous exceptional event of 18-20 November 2013, in which under relatively small eruptive magnetic flux $\Phi \approx 133 \times 10^{20} \mathrm{Mx}$ the GMS intensity reached $\mathrm{Ap} \approx 300$ (this storm was the 


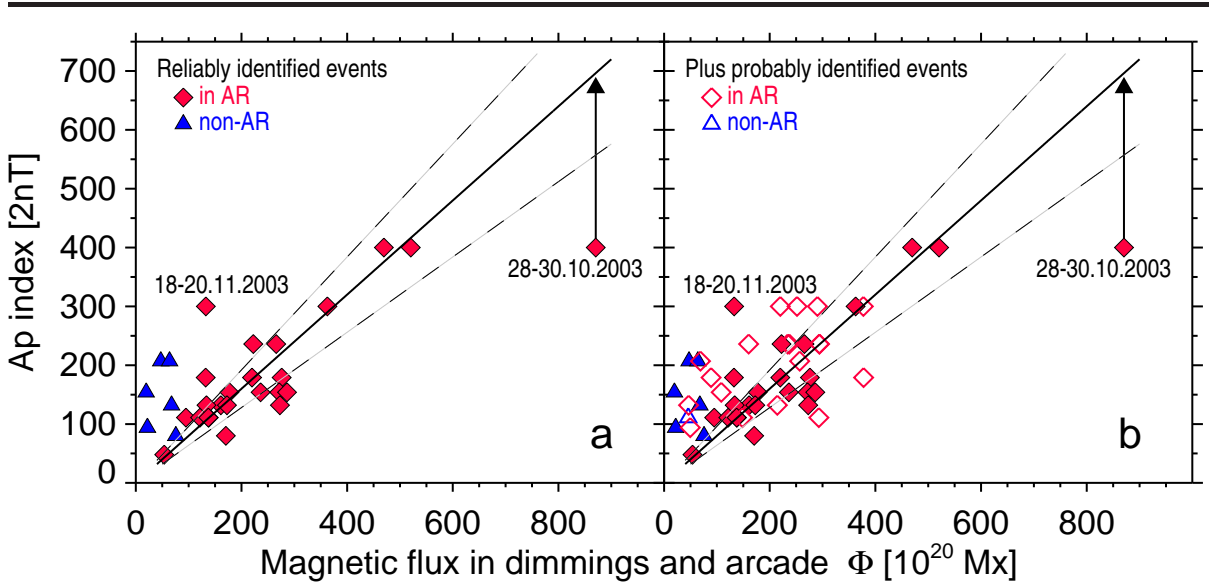

Figure 1. Dependence of the geomagnetic Ap index on the total magnetic flux in the arcades and dimming regions $\Phi$ : (a) for GMSs reliably identified with a definite solar eruption (filled symbols) and (b) for all considered events including those with probable solar source identification (open symbols). Red diamonds denote eruptions in ARs, and blue triangles denote eruptions of filaments outside ARs. The dashed lines delimit the adopted $20 \%$ deviation band.

strongest in Solar Cycle 23 in terms of the index Dst $\approx-422 \mathrm{nT}$ ). The causes of the exceptional properties of this extreme event are analyzed in detail by Grechnev et al. (2014). They, in particular, showed that the GMS was caused by arrival to the Earth of a compact spheromak with a magnetic field strength up to $|B| \approx 56 \mathrm{nT}$ and a large, prolonged south component $B_{z} \approx-46 \mathrm{nT}$. Such a strong field in the interplanetary magnetic cloud was preserved due to its anomalously weak expansion in the propagation from the Sun to the Earth. Below we do not consider this event because of its atypical properties.

The remaining reliably identified AR events, shown in Figure 1a, demonstrate a significant relation between the eruptive flux $\Phi$ and the geomagnetic index Ap. When the flux $\Phi$ increases from tens to about 500 (in $10^{20} \mathrm{Mx}$ units), the Ap index rises up to the formally maximum value of 400 . Three events with the largest eruptive flux indicate this dependence at the formal peak level $\mathrm{Ap}=$ 400. Real values of the Ap index for these three events are not given in catalogs. We discard temporarily the 28-30 October 2003 event with the largest magnetic flux $\Phi$ and assume that for two events with the magnetic flux $\Phi$ in a range of $(470-520) \times 10^{20} \mathrm{Mx}$ the formal index $\mathrm{Ap}=400$ was close to the real one. Then the dependence of the Ap index on the eruptive magnetic flux $\Phi$ for the AR events can be expressed by a linear relation $\mathrm{Ap}=0.8 \Phi$ (here again $\Phi$ is expressed in units of $10^{20} \mathrm{Mx}$ ). In this case, the correlation coefficient between the observed and calculated values of Ap reaches $r \approx 0.90$. For additional evaluation of the scatter in data points, we choose a deviation band bounded by \pm 0.2 from the regression line that appears to be acceptable for the forecasting. Calculations show that 14 out of 22 events (i.e., 64\%) fall into this deviation band.

Extrapolation of the obtained relation allows us to estimate a probable increase of the real Ap index for eruptions with the largest magnetic flux without the formal upper limit of $\mathrm{Ap}=400$. In particular, for the 28-30 October 2003 event with $\Phi \approx 870 \times 10^{20} \mathrm{Mx}$, the Ap index, in accordance with this dependence, 
should be around 700 . This estimate does not contradict the largest variations of the horizontal component of the Earth's magnetic field (about 1700-2500 nT) registered by high-latitude magnetometers (e.g., Panasyuk et al., 2004). According to the data of the magnetic observatory Moscow (IZMIRAN), in the period 21-24 UT on 29 October 2003 the field variations were about $1200 \mathrm{nT}$, that yields $\mathrm{Ap} \approx 800$. This also agrees well with the dependence presented above.

The dependence of the geomagnetic Ap index on the eruptive magnetic flux $\Phi$ for the AR events appears to be basically the same, when the GMSs with a probable source identification (open symbols in Figure $1 \mathrm{~b}$ ) are added to the unambiguously identified ones. Here, as expected, the scatter of the points increases, and the correlation coefficient between $\Phi$ and Ap reduces to $r \approx 0.73$. In this case, 19 points out of 41 (i.e., $46 \%$ ) fall into the same deviation band.

\section{Conclusion}

Our analysis has shown that for AR events a noticeable statistical relationship exists between the GMS intensity measured by the Ap index and the eruptive magnetic flux $\Phi$ in solar EUV arcades and dimming regions. This relationship also demonstrates that solar eruptions with the greatest magnetic flux can lead to the strongest GMSs, in which a real Ap index significantly exceeds the formally introduced maximum level of $\mathrm{Ap}=400$.

This result is consistent with those of the paper by Chertok et al. (2013), in which the dependencies were established on the same eruptive magnetic flux, $\Phi$, for such important space weather parameters as: i) the intensity of GMSs characterized by another geomagnetic index Dst, ii) the values of the Forbush decreases (FDs) of the galactic cosmic ray flux, and iii) the onset and peak times of these disturbances. This means that, in spite of many factors affecting the propagation of interplanetary clouds from the Sun to the Earth and the character of their interaction with the Earth's magnetosphere, the parameters of non-recurrent GMSs (and FDs) caused by CMEs/ICMEs are largely determined by the power of solar eruptions (in terms of the total magnetic flux in dimmings and arcades). This is especially conspicuous for sufficiently large eruptions. Just due to this fact, the statistical relationships exist between the magnetic flux in the source region of a CME, on the one hand, and the main parameters of GMSs and FDs, on the other hand.

The positive results of the present work and the analysis of Chertok et al. (2013) demonstrate the relevance of the of the magnetic flux in arcades and dimmings as a diagnostic parameter. This parameter can be used for the earliest estimations of the intensity and temporal characteristics of GMSs (and FDs), including both Dst and Ap indices, even without taking into account the information on associated CMEs and factors determining the $B_{z}$ component. At the present stage, we have considered only sufficiently intense GMSs. Therefore, when the GMS amplitude is estimated on the basis of the dependencies established in this article and by Chertok et al. (2013), it refers to a value close to the maximum that can be expected, provided that an ICME contains a significant negative (south) $B_{z}$ component. The proposed tool for the early diagnostics of 
the geoeffectiveness of a solar eruption should be considered and used as an initial component of a wider complex of methods of the short-term GMS and FD forecasting including also those based on the measurements of near-theSun CMEs, MHD models, stereoscopic observations of ICME propagation, and others.

Acknowledgements We are grateful to an anonymous reviewer for constructive comments, which helped us to improve the manuscript. The authors thank the SOHO EIT and MDI teams as well as the CDAW participants for data and materials used in the present study. $\mathrm{SOHO}$ is a project of international cooperation between ESA and NASA. This research was supported by the Russian Foundation of Basic Research under grants 12-02-00037, 14-0200367, and the Ministry of education and science of Russian Federation under projects 8407 and 14.518.11.7047.

\section{References}

Attrill, G., Nakwacki, M.S., Harra, L.K., van Driel-Gesztelyi, L., Mandrini, C.H., Dasso, S., Wang, J.: 2006, Solar Phys. 238, 117.

Bothmer V., Zhukov A.: 2007, In: Bothmer, V., Daglis, I.A. (eds.) Space Weather - Physics and Effects, 31.

Chertok, I.M., Grechnev, V.V.: 2005, Solar Phys. 229, 95.

Chertok, I.M., Grechnev, V.V.: 2006, Bull. Russsian Academy of Sci., Phys. 70, 1717.

Chertok, I.M., Grechnev, V.V., Belov, A.V., Abunin, A.A.: 2013, Solar Phys. 282, 175.

Delaboudinière, J.-P., Artzner, G.E., Brunaud, J., Gabriel, A.H., Hochedez, J.F., Millier, F., et al.: 1995, Solar Phys. 162, 291.

Domingo, V., Fleck, B., Poland, A.I.: 1995, Solar Phys. 162, 1.

Gopalswamy, N.: 2009, In: Tsuda, T., Fujii, R., Shibata, K., Geller, M.A. (eds.) Climate and Weather of the Sun-Earth Syst. (CAWSES): Selected Papers from the 2007 Kyoto Symp., 77 .

Grechnev, V.V., Uralov, A.M., Chertok, I.M., Belov, A.V., Filippov, B.P., Slemzin, V.A., Jackson, B.V.: 2014, Solar Phys. in press. DOI:10.1007/s11207-014-0596-5.

Hu, Q., Qiu, J., Dasgupta, B., Khare, A., Webb, G.M.: 2014, Astrophys. J. 793, article id. 53.

Hudson, H.S., Cliver, E.W.: 2001, J. Geophys. Res. 106, 25199.

Kahler, S.: 1977, Astrophys. J. 214, 891.

Kim, R.-S., Cho, K.-S, Moon, Y.-J., Dryer, M., Lee, J., Yi, Y., et al.: 2010, J. Geophys. Res. 115, A12108.

Leamon, R.J., Canfield, R.C., Jones, S.L., Lambkin, K., Lundberg, B.J., Pevtsov, A.A.: 2004, J. Geophys. Res. 109, 5106.

Mandrini, C.H., Pohjolainen, S., Dasso, S., Green, L.M., Démoulin, P., van Driel-Gesztelyi, L., Copperwheat, C., Foley, C.: 2005, Astron. Astrophys. 434, 725.

Panasyuk, M.I., Kuznetsov, S.N., Lazutin, L.L., Avdyushin, S.I., Alexeev, I.I., Ammosov, P.P. et al.: 2004, Cosmic Res. 42, 489

Qiu, J., Hu, Q., Howard, T.A., Yurchyshyn, V.B.: 2007, Astrophys. J. 659, 758.

Qiu, J., Yurchyshyn, V.B.: 2005, Astrophys. J. Lett. 634, L121.

Scherrer, P.H., Bogart, R.S., Bush, R.I., Hoeksema, J.T., Kosovichev, A.G., Schou, J., et al.: 1995, Solar Phys. 162, 129.

Siebert, M., Meyer, J.: 1996, In: Dieminger, W., Hartmann, G.K., Leitinger, R. (eds.) The Upper Atmosphere, 887.

Sterling, A.C., Hudson, H.S., Thompson, B.J., Zarro, D.: 2000, Astrophys. J. 532, 628.

Švestka, Z.: 2001, Space Sci. Rev. 95, 135.

Thompson, B.J., Plunkett, S.P., Gurman, J.B., Newmark, J.S., St. Cyr, O.C., Michels, D.J.: 1998, Geophys. Res. Lett. 25, 2465.

Tripathi, D., Bothmer, V., Cremades, H.: 2004, Astron. Astrophys. 422, 337.

Webb, D.F., Lepping, R.P., Burlaga, L.F., DeForest, C.E., Larson, D.E., Martin, S.F., et al.: 2000, J. Geophys. Res. 105, 27251. 
Zabolotnaya, N.A.: 2007, Indices of Geomagnetic Activity, LKI, Moscow. (in Russian).

Zhang, J., Richardson, I.G., Webb, D.F., Gopalswamy, N., Huttunen, E., Kasper, J., et al.: 2007, J. Geophys. Res. 112, A10102. 